\title{
Age related gene DST represents an independent prognostic factor for MYCN non-amplified neuroblastoma
}

\author{
Haiwei Wang ${ }^{1 * \dagger}$, Xinrui Wang ${ }^{1 \dagger}$, Liangpu Xu', Ji Zhang ${ }^{2^{*}}$ and Hua Cao ${ }^{1 *}$
}

\begin{abstract}
Background: MYCN amplification and age are two critical prognostic factors of pediatric neuroblastoma. Previously, we had revealed the prognosis of MYCN target genes. However, the prognostic effects of age related genes in neuroblastoma are unclear.

Methods: The prognostic significance of age and MYCN amplification was determined through multivariate cox regression and Kaplan-Meier survival analysis. Genes differentially expressed in MYCN non-amplified younger neuroblastoma patients were identified using Therapeutically Applicable Research to Generate Effective Treatments (TARGET) and Gene Expression Omnibus (GEO) datasets. The prognostic effects of age related genes ALCAM, CACN A2D3, DST, EPB41L4A and KIF1B in pediatric neuroblastoma patients were determined by Kaplan-Meier survival.

Results: In a pediatric pan-cancer analysis, age was associated with the overall survival of pediatric B-lineage acute lymphoblastic leukemia, neuroblastoma and wilms tumor in TARGET dataset. Moreover, the prognostic effects of age in neuroblastoma were validated using two independent neuroblastoma cohorts. Furthermore, age and MYCN amplification were independent prognostic factors in pediatric neuroblastoma. Compared with MYCN nonamplified older neuroblastoma patients, MYCN non-amplified younger neuroblastoma patients had better clinical outcomes. ALCAM, CACNA2D3, DST, EPB41L4A and KIF1B were highly expressed in MYCN non-amplified younger neuroblastoma patients. And the higher expression levels of ALCAM, CACNA2D3, DST, EPB41L4A or KIF1B were associated with better prognosis of MYCN non-amplified neuroblastoma patients. DST was an independent prognostic factor in MYCN non-amplified neuroblastoma patients and MYCN non-amplified neuroblastoma younger patients with higher DST expression levels had the best clinical overall survival.
\end{abstract}

Conclusions: Age related gene DST was an independent prognostic factor in MYCN non-amplified neuroblastoma. MYCN non-amplified younger neuroblastoma patients with higher DST expression levels had the best clinical overall survival.

Keywords: Pediatric neuroblastoma, MYCN amplification, Age, DST, TARGET, GEO

\footnotetext{
* Correspondence: hwwang@sibs.ac.cn; Zj11222@rjh.com.cn;

caohua69@fjmu.edu.cn

†Haiwei Wang and Xinrui Wang contributed equally to this work.

${ }^{1}$ Medical Research Center, Fujian Maternity and Child Health Hospital,

Affiliated Hospital of Fujian Medical University, Fuzhou, Fujian, China

${ }^{2}$ State Key Laboratory for Medical Genomics, Shanghai Institute of

Hematology, Rui-Jin Hospital Affiliated to School of Medicine, Shanghai Jiao

Tong University, Shanghai, China
}

C The Author(s). 2021 Open Access This article is licensed under a Creative Commons Attribution 4.0 International License, which permits use, sharing, adaptation, distribution and reproduction in any medium or format, as long as you give appropriate credit to the original author(s) and the source, provide a link to the Creative Commons licence, and indicate if changes were made. The images or other third party material in this article are included in the article's Creative Commons licence, unless indicated otherwise in a credit line to the material. If material is not included in the article's Creative Commons licence and your intended use is not permitted by statutory regulation or exceeds the permitted use, you will need to obtain permission directly from the copyright holder. To view a copy of this licence, visit http://creativecommons.org/licenses/by/4.0/. The Creative Commons Public Domain Dedication waiver (http://creativecommons.org/publicdomain/zero/1.0/) applies to the data made available in this article, unless otherwise stated in a credit line to the data. 


\section{Background}

Pediatric neuroblastoma is a heterogeneous disease with distinctive clinical outcomes [1, 2]. MYCN amplification is an important prognostic factor in neuroblastoma [3]. Pediatric neuroblastoma patients with MYCN amplification are associated with poor prognosis [4-6]. MYCN belongs to the MYC transcription factor family and regulates the expression levels of multiple target genes [7]. Independent of $\mathrm{MYCN}$ amplification, MYCN expression levels [8] and MYCN regulated signature [9] are also correlated with the unfavorable outcomes of neuroblastoma. Using published gene expression datasets, previously, we had identified six MYCN target genes which could be used to predict the clinical outcomes of neuroblastoma [10]. Moreover, MYCN amplification represents a therapeutic target in neuroblastoma. Bromodomain inhibitors have specific efficacy in MYCN amplified neuroblastoma [11-13].

Besides MYCN amplification, age at initial neuroblastoma diagnosis is also a prognostic factor [14]. Compared with younger neuroblastoma patients, older neuroblastoma patients usually had worse prognostic outcomes [15]. However, the relationships of age and MYCN amplification in the predication of the clinical outcomes of neuroblastoma are unclear. Also, the transcriptional profiling associated with age and $\mathrm{MYCN}$ amplification is unknown.

Here, using TARGET [16], GSE49710 [17] and GSE85047 datasets [18], the transcriptional profiling of MYCN non-amplified younger neuroblastoma patients was identified. Also, the prognostic effects of age and MYCN amplification related genes in neuroblastoma were determined. Our analysis revealed that MYCN non-amplified neuroblastoma was heterogeneous and could be divided into sub-groups based on the age at initial neuroblastoma diagnosis or the expression levels of DST. MYCN non-amplified younger patients with higher DST expression levels had the best clinical overall survival in neuroblastoma.

\section{Methods}

\section{Data collection}

The TARGET datasets were downloaded from https:// ocg.cancer.gov/. The GSE49710 and GSE85047 datasets were downloaded from https://www.ncbi.nlm.nih.gov/ geo/. All the data was further processed using R software (version 3.5.0; https://www.r-project.org/).

\section{Prognostic effects of age and MYCN amplification}

Neuroblastoma patients were divided into older and younger groups based on the mean age at initial diagnosis. The mean age of neuroblastoma patients at initial diagnosis in TARGET, GSE49710 or GSE85047 dataset was 2.5 years old, 2.1 years old or 2.2 years old, respectively. Kaplan-Meier estimator in GraphPad Prism software (version 5.0; https://www.graphpad.com/) was applied to determine the different clinical overall survival of neuroblastoma patients with different age or MYCN amplification status using TARGET, GSE49710 and GSE85047 datasets. $P$ values were determined by Log-rank test.

\begin{abstract}
Heatmap
The differentially expressed genes in MYCN nonamplified younger neuroblastoma patients were presented using heatmaps by R software "pheatmap" package (version 1.0.12; https://cran.r-project.org/web/ packages/pheatmap/ index.html).
\end{abstract}

\section{Venn diagram}

The common genes which were differentially expressed in MYCN non-amplified younger neuroblastoma in TARGET, GSE49710 and GSE85047 datasets were determined by VENNY 2.1 software (http://bioinfogp.cnb. csic.es/tools/venny/index.html).

\section{Kyoto encyclopedia of gens and genomes (KEGG) signaling pathway and transcription factors enrichment analysis}

MYCN non-amplified younger neuroblastoma associated KEGG signaling pathways [19-21] and transcription factors were determined using The Database for Annotation, Visualization and Integrated Discovery (DAVID) website (version 6.8; https://david.ncifcrf.gov) [22, 23].

\section{Prognostic effects of ALCAM, CACNA2D3, DST, EPB41L4A and KIF1B}

Neuroblastoma patients were divided into two subgroups based on the mean expression levels of ALCAM, CACNA2D3, DST, EPB41L4A or KIF1B. R software 'survival' package (version 3.1-8; https://cran.r-project.org/ web/packages/survival/ index.html) was used to test the clinical overall survival of neuroblastoma with higher expression levels or lower expression levels of ALCAM, CACNA2D3, DST, EPB41L4A and KIF1B. Log-rank test was used to determine the $P$ values.

\section{Univariate and multivariate cox regression}

The prognosis of age in different pediatric tumor types was determined by univariate cox regression analysis. The associations of ALCAM, CACNA2D3, DST, EPB41L4A and KIF1B in MYCN non-amplified neuroblastoma patients were tested by multivariate cox regression analysis using $\mathrm{R}$ software 'survival' package 'coxph' method (version 3.1-8). 


\section{Correlation plot}

R software 'corrplot' package (version 0.84; https://cran. r-project.org/web/packages/corrplot/index.html) was used to determine the correlation of ALCAM, CACN A2D3, DST, EPB41L4A and KIF1B based on their expression levels in TARGET, GSE49710 and GSE85047 datasets.

\section{Statistical analysis}

The box plots were generated from GraphPad Prism software. Statistical analysis was performed using the two tails paired Student's $t$ test in $\mathrm{R}$ software. $P$ value less than 0.05 was chosen to be statistically significant difference.

\section{Results}

Age is associated with the overall survival of pediatric neuroblastoma patients

Previously, we revealed the prognostic effects of age in each adult tumor type using The Cancer Genome Atlas (TCGA) datasets [24]. Here, using TARGET datasets generated from St Jude Children's Research Hospital, the prognostic effects of age in each pediatric tumor type were further analyzed. Collectively, 2419 patients from six pediatric tumor types were used for further studies. Although, those six tumor types were all developed in children or adolescents, the mean age was quite different (Fig. 1a). The mean age of neuroblastoma patients was 2.5 years old, much younger than other patients. And osteosarcoma represented the most old pediatric cancer type (Fig. 1a).

First, using univariate cox regression analysis, we found that age was significantly associated with the overall survival in pediatric B-lineage acute lymphoblastic leukemia, neuroblastoma and wilms tumor patients (Fig. 1a). However, age had no prognostic effect in pediatric acute myeloid leukemia, T-lineage acute lymphoblastic leukemia or osteosarcoma patients (Fig. 1a).

Similar results were obtained through the KaplanMeier survival analysis that age was a critical prognostic factor for pediatric B-lineage acute lymphoblastic leukemia, neuroblastoma and wilms tumor (Fig. 1b). Particularly, age was most associated with the overall survival of pediatric B-lineage acute lymphoblastic leukemia patients $(P<0.0001$ and $H R=5.81)$ (Fig. 1b). However, the overall survival of older acute myeloid leukemia, T-lineage acute lymphoblastic leukemia or osteosarcoma patients was not significantly different from younger patients (Fig. 1b).

Age and MYCN amplification are independent prognostic factors in pediatric neuroblastoma

The prognostic effects of age in pediatric neuroblastoma were further confirmed using 498 Chinese primary neuroblastomas deposited in GSE49710 dataset and 283 primary neuroblastomas deposited in GSE85047 dataset assembled by the European Neuroblastoma Research Consortium (NRC). The mean age of neuroblastoma patients in GSE49710 and GSE85047 datasets was 2.1 and 2.2 years old, respectively. Similar to the results derived from TARGET dataset, we found that, compared with younger pediatric neuroblastoma, older pediatric neuroblastoma patients had worse clinical outcomes in both GSE49710 and GSE85047 datasets (Fig. 2a).

MYCN amplification was also associated with the clinical outcomes of pediatric neuroblastoma [5]. So, next, we determined the relationships of age and MYCN amplification in neuroblastoma. We found that there was no significant difference of the mean age in pediatric neuroblastoma patients with or without MYCN amplification in TARGET and GSE85047 datasets (Fig. 2b). However, compared with neuroblastoma patients without MYCN amplification, the mean age in MYCN amplified neuroblastoma patients was higher in GSE49710 dataset (Fig. 2b). Furthermore, the multivariate cox regression assay suggested that age and MYCN amplification were independent prognostic factors in pediatric neuroblastoma in TARGET, GSE49710 and GSE85047 datasets (Fig. 2c).

\section{MYCN non-amplified younger neuroblastoma patients have better prognosis}

Next, we determined the combination of age and MYCN amplification in the predication of the clinical outcomes of pediatric neuroblastoma. Pediatric neuroblastoma patients in TARGET, GSE49710 and GSE85047 datasets were divided into four sub-groups based on the mean age and MYCN amplification status. The Kaplan-Meier plots showed that MYCN amplified older patients and MYCN amplified younger patients had not significantly different clinical outcomes (Fig. 3). However, MYCN non-amplified younger neuroblastoma patients had significantly favorable prognosis than MYCN non-amplified older neuroblastoma patients in TARGET, GSE49710 and GSE85047 datasets (Fig. 3). Those results suggested that MYCN non-amplified pediatric neuroblastoma was heterogeneous, and could be divided into older and younger pediatric neuroblastoma sub-groups.

\section{Identification of the differentially expressed genes in MYCN non-amplified younger neuroblastoma patients} Moreover, we tried to identify the differentially expressed genes in MYCN non-amplified younger neuroblastoma patients in TARGET dataset. Compared with MYCN amplified neuroblastoma patients and MYCN non-amplified older neuroblastoma patients, 64 


\begin{tabular}{cccccccc}
\hline Tumor type & & & & & & \\
\cline { 2 - 7 } & & $\begin{array}{c}\text { Mean age } \\
\text { (Years) }\end{array}$ & $\begin{array}{c}\text { Younger } \\
\text { (Number) }\end{array}$ & $\begin{array}{c}\text { Older } \\
\text { (Number) }\end{array}$ & HR & P value & $95 \%$ Cl \\
\hline Acute Myeloid Leukemia & $9.6 \pm 0.3$ & 392 & 285 & 0.99 & 0.61 & $0.97-1.01$ \\
B-lineage Acute Lymphoblastic Leukemia & $6.4 \pm 0.2$ & 189 & 309 & 1.18 & $1.6 \mathrm{e}-08$ & $1.12-1.26$ \\
T-lineage Acute Lymphoblastic Leukemia & $9.5 \pm 0.4$ & 69 & 76 & 1.01 & 0.91 & $0.89-1.14$ \\
Neuroblastoma & $2.5 \pm 0.12$ & 188 & 311 & 1.08 & 0.0026 & $1.21-1.36$ \\
Osteosarcoma & $16.9 \pm 0.9$ & 42 & 95 & 1 & 0.81 & $0.96-1.57$ \\
Wilms Tumor & $3.8 \pm 0.11$ & 234 & 293 & 1.04 & $1.4 \mathrm{e}-11$ & $1.03-1.06$ \\
\hline
\end{tabular}

b

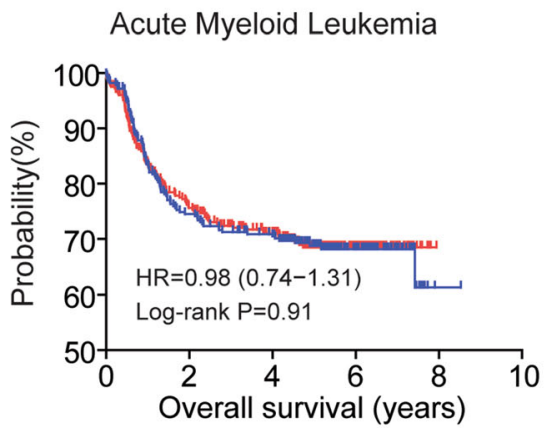
Acute Lymphoblastic Leukemia

T-lineage

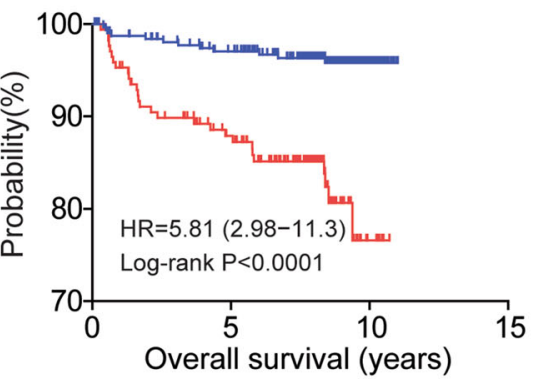

Acute Lymphoblastic Leukemia

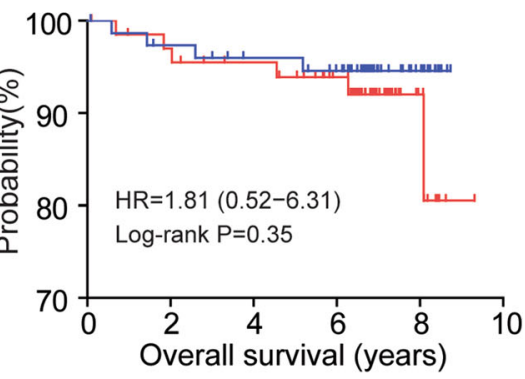

Neuroblastoma

Osteosarcoma

Wilms Tumor
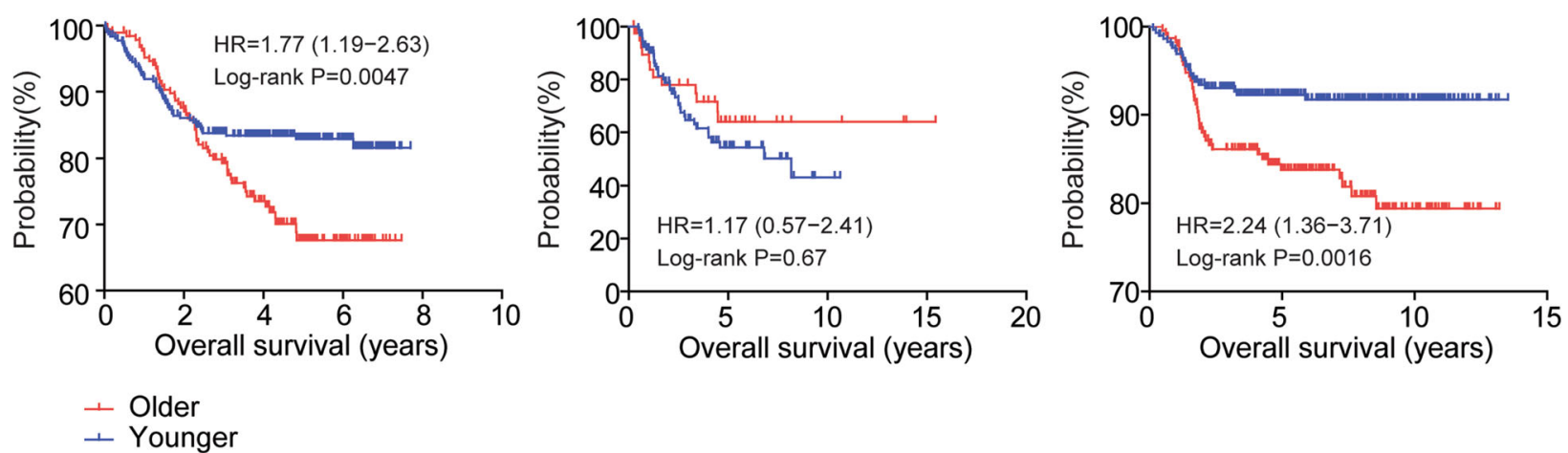

Fig. 1 Age is associated with the overall survival of pediatric neuroblastoma patients. a Univariate cox regression was used to reveal the prognostic significance of age in different pediatric tumor types in TARGET dataset. $\mathbf{b}$ The Kaplan-Meier curves showed the different overall survival of older (red) and younger (blue) tumor patients in each pediatric tumor type using TARGET dataset. $P$ value was determined by Log-rank test. HR represented the hazard ratio

genes were highly expressed in MYCN non-amplified younger neuroblastoma patients in TARGET dataset (Fig. 4a). However, there were only 12 genes were downregulated in MYCN non-amplified younger neuroblastoma patients (Fig. 4a). Those differentially expressed genes were involved in the regulation of neuron projection, the regulation of GTPase activity and the regulation of cell-cell adhesion (Fig. 4b).

The differentially expressed genes in MYCN nonamplified younger neuroblastoma patients in GSE49710 and GSE85047 datasets were also identified. There were
2952 and 612 genes were differentially expressed in MYCN non-amplified younger neuroblastoma patients in GSE49710 and GSE85047 datasets, respectively (Fig. 4a). Among them, ALCAM, BTBD9, CACNA2D3, DST, EPB41L4A, FGD6, GMEB1, IGSF3 and KIF1B were commonly changed in MYCN non-amplified younger neuroblastoma patients in TARGET, GSE49710 and GSE85047 datasets (Fig. 4c). Using heatmaps, we showed that those genes were all over-expressed in MYCN non-amplified younger neuroblastoma patients (Fig. 4d). 
a

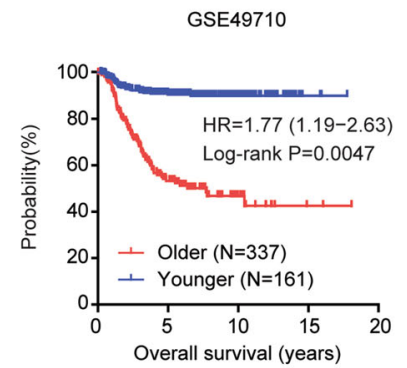

b

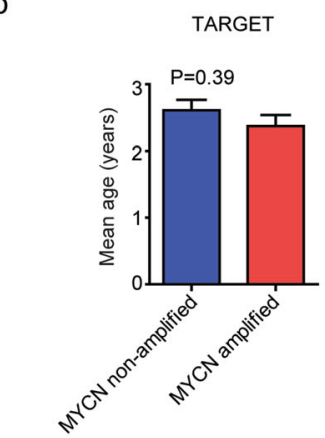

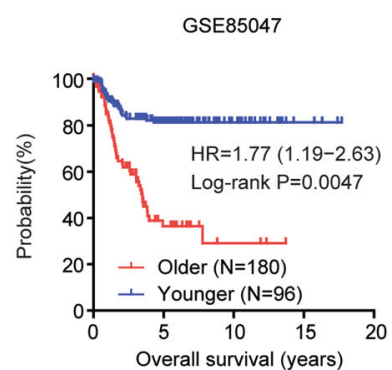

GSE49710

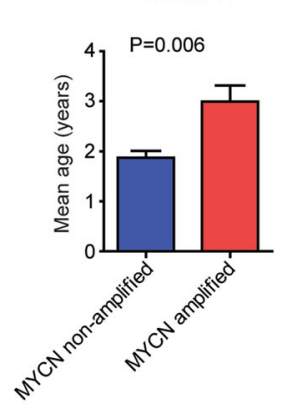

GSE85047

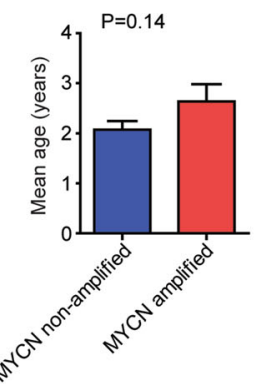

C

\begin{tabular}{ccccc}
\multicolumn{5}{c}{ TARGET } \\
\hline Variable & Coefficient & HR & P value & $95 \% \mathrm{Cl}$ \\
\hline Age & 0.08 & 1.08 & 0.0001 & $1.04-1.12$ \\
MYCN & -0.56 & 0.57 & $2.6 e-06$ & $0.45-0.72$ \\
\hline
\end{tabular}

\begin{tabular}{ccccc}
\multicolumn{5}{c}{ GSE49710 } \\
\hline Variable & Coefficient & HR & P value & $95 \% \mathrm{Cl}$ \\
\hline Age & 0.34 & 1 & $1.98 \mathrm{e}-12$ & $1-1.1$ \\
MYCN & 1.97 & 7.2 & $2 \mathrm{e}-16$ & $4.9-10.6$ \\
\hline
\end{tabular}

GSE85047

\begin{tabular}{ccccc}
\hline Variable & Coefficient & HR & P value & $95 \% \mathrm{Cl}$ \\
\hline Age & 0.29 & 1 & 0.0002 & $1-1.1$ \\
MYCN & 1.69 & 5.4 & $1.24 \mathrm{e}-12$ & $3.4-8.6$ \\
\hline
\end{tabular}

Fig. 2 Age and MYCN amplification are independent prognostic factors in pediatric neuroblastoma. a Overall survival was analyzed in older (red) and younger (blue) pediatric neuroblastoma patients in GSE49710 and GSE85047 datasets. P values were generated from Log-rank test. b Box plot showed the mean age of MYCN amplified (red) and MYCN non-amplified (blue) pediatric neuroblastoma patients. P values were determined using the Student's t test. c Multivariate cox regression was used to test the prognostic relevance of age and MYCN amplification in neuroblastoma, using TARGET, GSE49710 and GSE85047 datasets

High expression levels of ALCAM, CACNA2D3, DST, EPB41L4A or KIF1B are associated with the favorable prognosis of MYCN non-amplified neuroblastoma patients

Furthermore, we determined the prognostic effects of ALCAM, BTBD9, CACNA2D3, DST, EPB41L4A, FGD6, GMEB1, IGSF3 and KIF1B in MYCN non-amplified neuroblastoma patients. We found that ALCAM, CACN A2D3, DST, EPB41L4A and KIF1B were associated with the favorable prognosis of MYCN non-amplified neuroblastoma patients. MYCN non-amplified neuroblastoma patients with lower expression levels of ALCAM, CACN A2D3, DST, EPB41L4A or KIF1B were with lower overall survival in TARGET dataset (Fig. 5a), GSE49710 dataset (Fig. 5b) and GSE85047 dataset (Fig. 5c).
Interestingly, high expression levels of ALCAM, CACNA2D3, DST, EPB41L4A or KIF1B were not only associated with the prognosis of $\mathrm{MYCN}$ non-amplified neuroblastoma patients, but also were associated with the prognosis of all neuroblastoma patients. Neuroblastoma patients with higher expression levels of ALCAM, CACNA2D3, DST, EPB41L4A or KIF1B had better clinical overall survival in TARGET dataset (Fig. 6a), GSE49710 dataset (Fig. 6b) and GSE85047 dataset (Fig. 6c).

\section{Expression level of DST is an independent prognostic} factor in MYCN non-amplified pediatric neuroblastoma Next, we tried to determine the associations of ALCAM, CACNA2D3, DST, EPB41L4A and KIF1B in MYCN 
TARGET

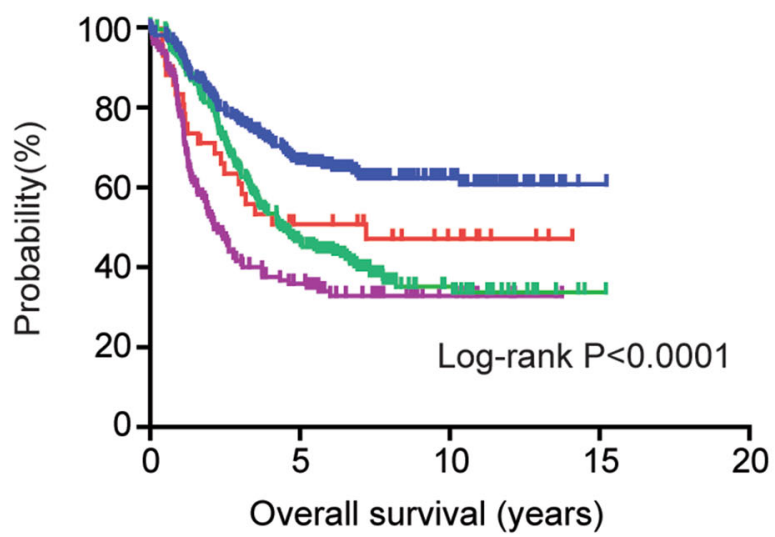

GSE85047

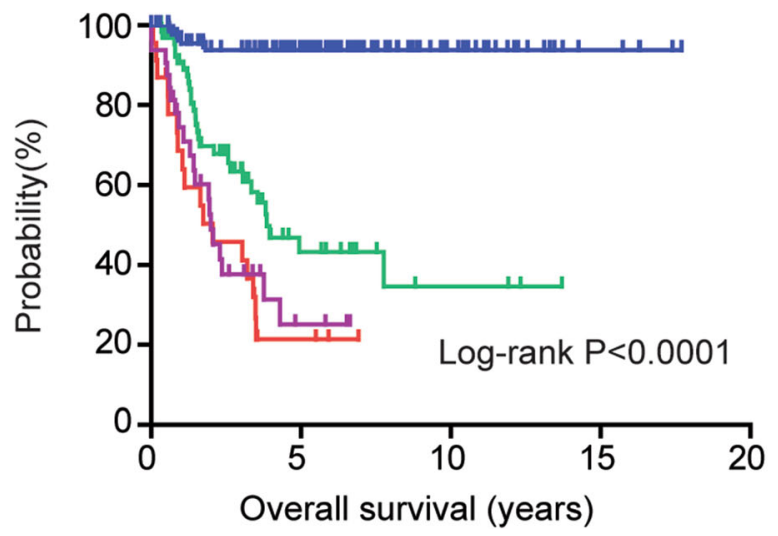

GSE49710

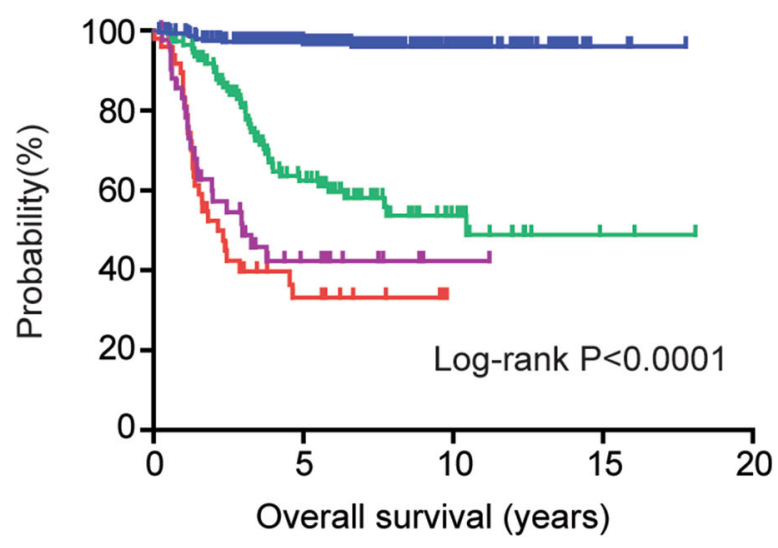

$\perp$ MYCN non-amplified and young

$\perp$ MYCN non-amplified and old

$\perp$ MYCN amplified and young

$\perp$ MYCN amplified and old

Fig. 3 MYCN non-amplified younger neuroblastoma patients have better prognosis. Pediatric neuroblastoma patients in TARGET, GSE49710 and GSE85047 datasets were divided into MYCN amplified older, MYCN amplified younger, MYCN non-amplified older and MYCN non-amplified younger four sub-groups. The Kaplan-Meier plots showed the different overall survival of those four sub-groups. P values were determined using Log-rank test. HR represented the hazard ratio

non-amplified neuroblastoma patients. Based on their expression levels in MYCN non-amplified neuroblastoma patients, we found those genes were highly correlated with each other, as demonstrated the high correlation coefficients of those genes in TARGET, GSE49710 and GSE85047 datasets (Fig. 7a). And ALCA M, DST, CACNA2D3 and KIF1B genes were all predicted to be the target genes of IRF1, FOXO3 or CEBPA transcription factors using DAVID analysis (Fig. 7b).

Furthermore, using multivariate cox regression, we determined the correlations of age, ALCAM, CACNA2D3, DST, EPB41L4A or KIF1B expression in the prediction of the clinical overall survival of MYCN non-amplified neuroblastoma patients. Age alone was an independent prognostic marker of MYCN non-amplified neuroblastoma in GSE49710 and GSE85047 datasets, but not in TARGET dataset (Fig. 7c). In TARGET, GSE49710 and GSE85047 datasets, we found that the expression level of DST was an independent prognostic marker (Fig. 7c). The expression level of EPB41L4A was an independent prognostic marker in GSE85047 dataset (Fig. 7c). Those results suggested that, although, ALCAM, CACNA2D3, DST, EPB41L4A and KIF1B shared similar expression signature, the prognostic significance of DST was independent.

\section{Younger patients with higher DST expression level have} the best prognosis in MYCN non-amplified pediatric neuroblastoma

Since age associated gene DST was an independent prognostic factor in MYCN non-amplified pediatric neuroblastoma, we wondered if the combination of DST, age and MYCN could achieve the best prognostic significance. To test this hypothesis, MYCN non-amplified pediatric neuroblastoma patients in TARGET dataset were divided into older patients with higher DST 


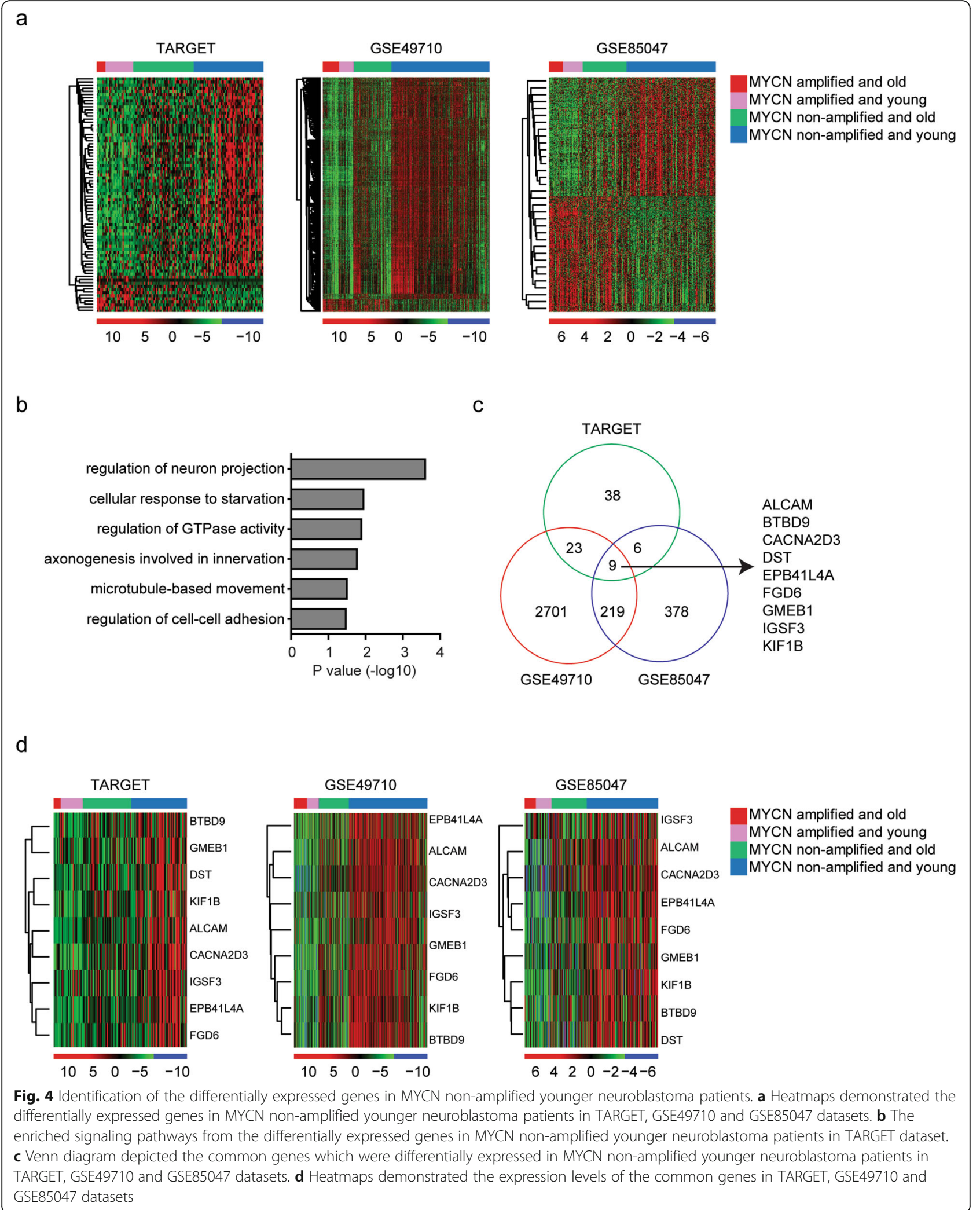


a

b

C
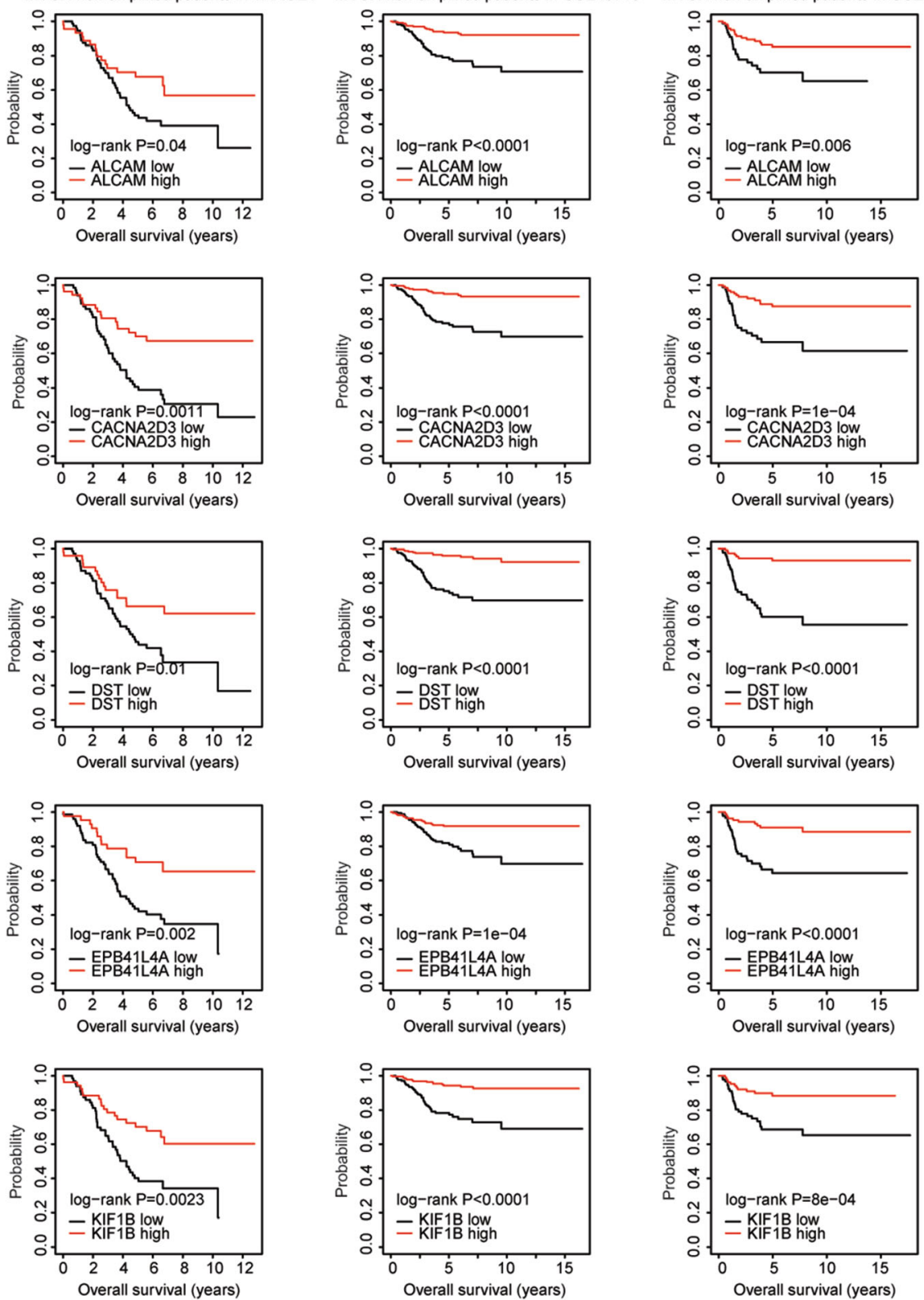

Fig. 5 High expression levels of ALCAM, CACNA2D3, DST, EPB41L4A or KIF1B are associated with the favorable prognosis of MYCN non-amplified neuroblastoma patients. a-c The Kaplan-Meier plots demonstrated the prognostic effects of ALCAM, CACNA2D3, DST, EPB41L4A and KIF1B in MYCN non-amplified pediatric neuroblastoma using TARGET dataset (a), GSE49710 dataset (b) and GSE85047 dataset (c). Patients were divided into two sub-groups based on the mean expression levels of ALCAM, CACNA2D3, DST, EPB41L4A or KIF1B. The log-rank test was used to determine the overall survival $P$ values

expression, older patients with lower DST expression, younger patients with higher DST expression and younger patients with lower DST expression four sub-groups. We found that MYCN non-amplified younger patients with higher DST expression had the best prognosis. However, other three sub-groups had similar prognosis in TARGET dataset (Fig. 8). In GSE49710 and GSE85047 datasets, MYCN non-amplified younger patients with higher DST expression also had the best prognosis, while, MYCN non-amplified older patients with lower DST expression had the worst prognosis (Fig. 8).

\section{Discussion}

Using the integrated analysis of TARGET, GSE49710 and GSE85047 datasets, our results showed that MYCN 
a
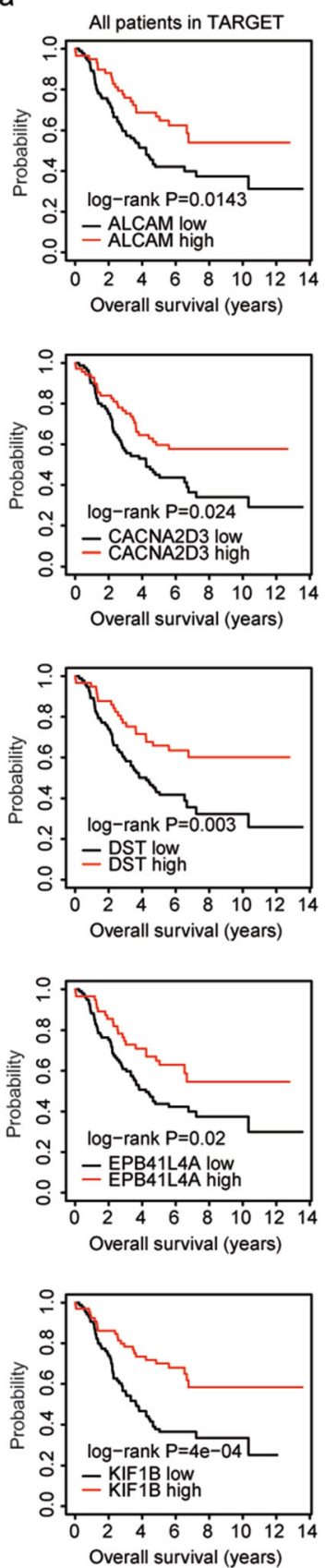

b
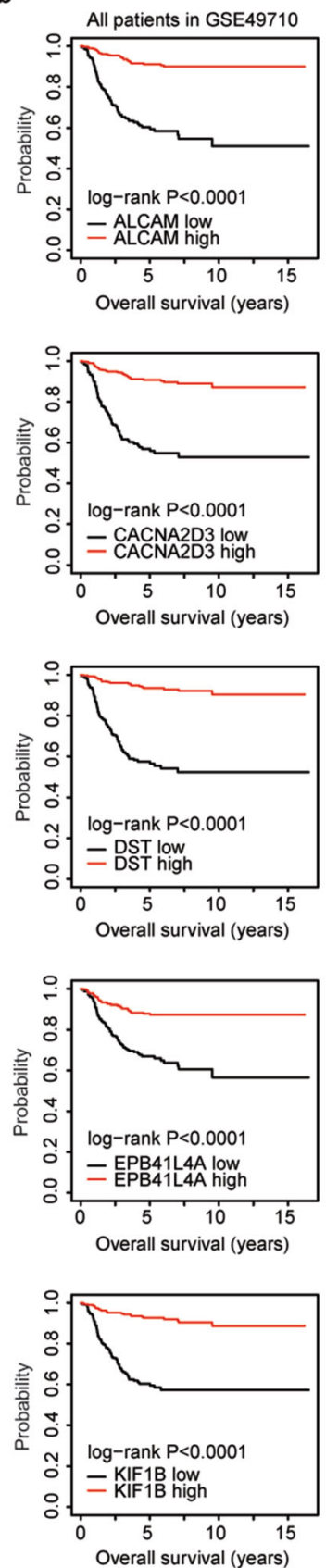

C
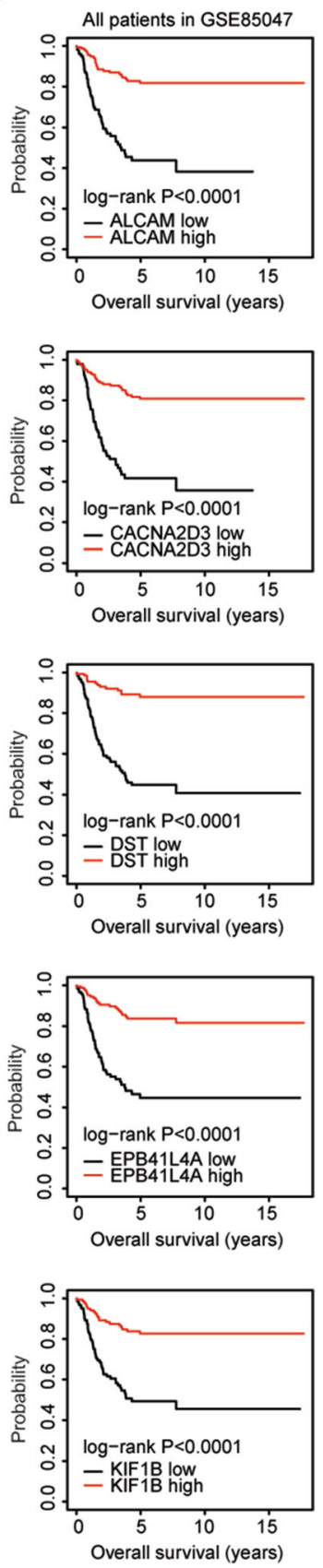

Fig. 6 High expression levels of ALCAM, CACNA2D3, DST, EPB41L4A or KIF1B are associated with the favorable prognosis of neuroblastoma patients. The Kaplan-Meier plots demonstrated the prognostic effects of ALCAM, CACNA2D3, DST, EPB41L4A or KIF1B in pediatric neuroblastoma using TARGET dataset (a), GSE49710 dataset (b) and GSE85047 dataset (c)

non-amplified pediatric neuroblastoma was heterogeneous, comprised younger and older sub-groups. And MYCN non-amplified younger pediatric neuroblastoma patients had better prognosis. Furthermore, MYCN nonamplified younger patients with higher DST expression levels had the best prognosis. On the contrary, MYCN non-amplified older patients with lower DST expression levels had worse prognosis. These analyses provided deep understanding of the heterogeneity of neuroblastoma.

Dystonin/BPAG1 (DST) is bullous pemphigoid antigen 1 , involving the autoimmune response in the development of bullous pemphigoid [25]. DST is also associated with multiple neurological disorders, including Parkinson's disease [26], Alzheimer's disease [27], epilepsy, dementia and multiple sclerosis [28], through regulation of 
a

MYCN non-amplified patients in TARGET

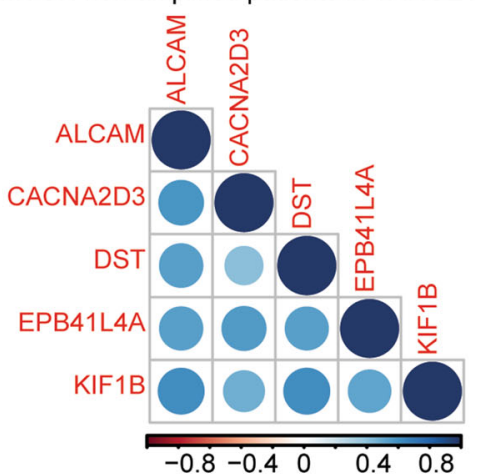

b

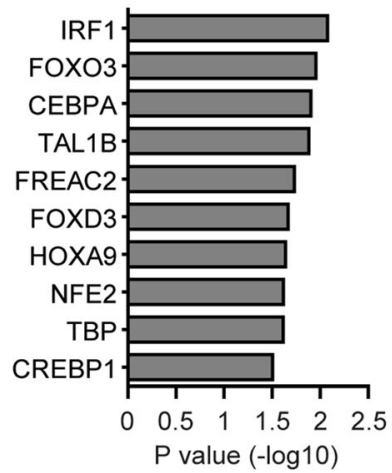

MYCN non-amplified patients in GSE49710

\begin{tabular}{ccccc}
\hline Variable & Coefficient & HR & P value & $95 \% \mathrm{Cl}$ \\
\hline Age & 0.1 & 1.1 & $6.5 \mathrm{e}-06$ & $1.06-1.16$ \\
ALCAM & -0.08 & 0.92 & 0.63 & $0.68-1.27$ \\
CACNA2D3 & -0.18 & 0.84 & 0.21 & $0.64-1.11$ \\
DST & -0.62 & 0.54 & 0.008 & $0.34-0.85$ \\
EPB41L4A & -0.04 & 0.96 & 0.82 & $0.68-1.53$ \\
KIF1B & -0.24 & 0.78 & 0.37 & $0.46-1.33$ \\
\hline
\end{tabular}

MYCN non-amplified patients in GSE49710

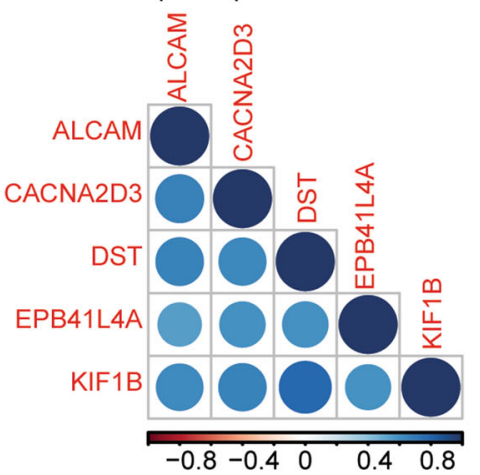

MYCN non-amplified patients in GSE85047

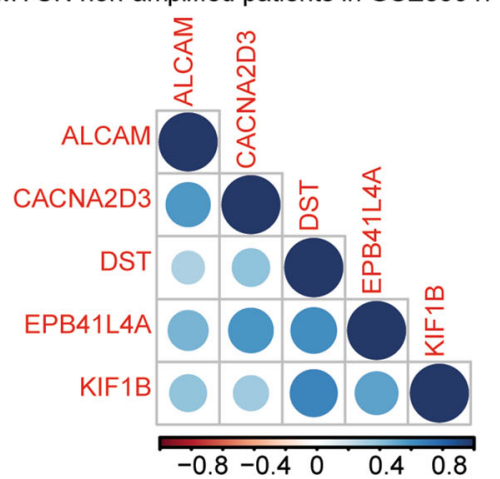

C

MYCN non-amplified patients in TARGET

\begin{tabular}{ccccc}
\hline Variable & Coefficient & HR & P value & $95 \% \mathrm{Cl}$ \\
\hline Age & 0.018 & 1.02 & 0.73 & $0.92-1.12$ \\
ALCAM & 0.001 & 1 & 0.71 & $0.99-1$ \\
CACNA2D3 & -0.012 & 0.99 & 0.17 & $0.97-1$ \\
DST & -0.009 & 0.99 & 0.013 & $0.98-1$ \\
EPB41L4A & -0.018 & 0.98 & 0.78 & $0.86-1.12$ \\
KIF1B & -0.025 & 0.98 & 0.24 & $0.94-1.02$ \\
\hline
\end{tabular}

MYCN non-amplified patients in GSE85047

\begin{tabular}{ccccc}
\hline Variable & Coefficient & HR & P value & $95 \% \mathrm{Cl}$ \\
\hline Age & 0.16 & 1.17 & 0.0003 & $1.08-1.28$ \\
ALCAM & 0.04 & 1.04 & 0.89 & $0.6-1.8$ \\
CACNA2D3 & 0.12 & 1.13 & 0.56 & $0.75-1.72$ \\
DST & -0.87 & 0.42 & 0.028 & $0.19-0.91$ \\
EPB41L4A & -0.79 & 0.45 & 0.003 & $0.27-0.77$ \\
KIF1B & 0.08 & 1.09 & 0.82 & $0.52-2.27$ \\
\hline
\end{tabular}

Fig. 7 Expression level of DST is an independent prognostic factor in MYCN non-amplified pediatric neuroblastoma. a Corrplots demonstrated the associations of ALCAM, CACNA2D3, DST, EPB41L4A and KIF1B in MYCN non-amplified neuroblastoma patients in TARGET, GSE49710 and GSE85047 datasets. The color of the circle represented the correlation coefficients. b The enriched transcription factors of ALCAM, CACNA2D3, DST, EPB41L4A and KIF1B genes. c Multivariate cox regression was used to determine the correlation of age, ALCAM, CACNA2D3, DST, EPB41L4A or KIF1B expression levels and overall survival in MYCN non-amplified neuroblastoma patients in TARGET, GSE49710 and GSE85047 datasets. HR represented hazard ratio

cytoskeletal dynamics [29] and cell migration [30]. However, the functions of DST in neuroblastoma are barely known. We found that DST was an independent prognostic factor in MYCN non-amplified pediatric neuroblastoma and highly expressed in MYCN non-amplified younger neuroblastoma patients. Combination of DST, age and MYCN achieved best prognostic effects in neuroblastoma. ALCAM, CACNA2D3, EPB41L4A and
KIF1B shared similar expression signature and prognostic effects with DST. Moreover, the prognostic significances of ALCAM, CACNA2D3, EPB41L4A and KIF1B in MYCN non-amplified pediatric neuroblastoma were not previously reported.

The purpose of this study was to determine the prognostic effects of age related genes in neuroblastoma. The present study suggested the relative heterogeneity of 


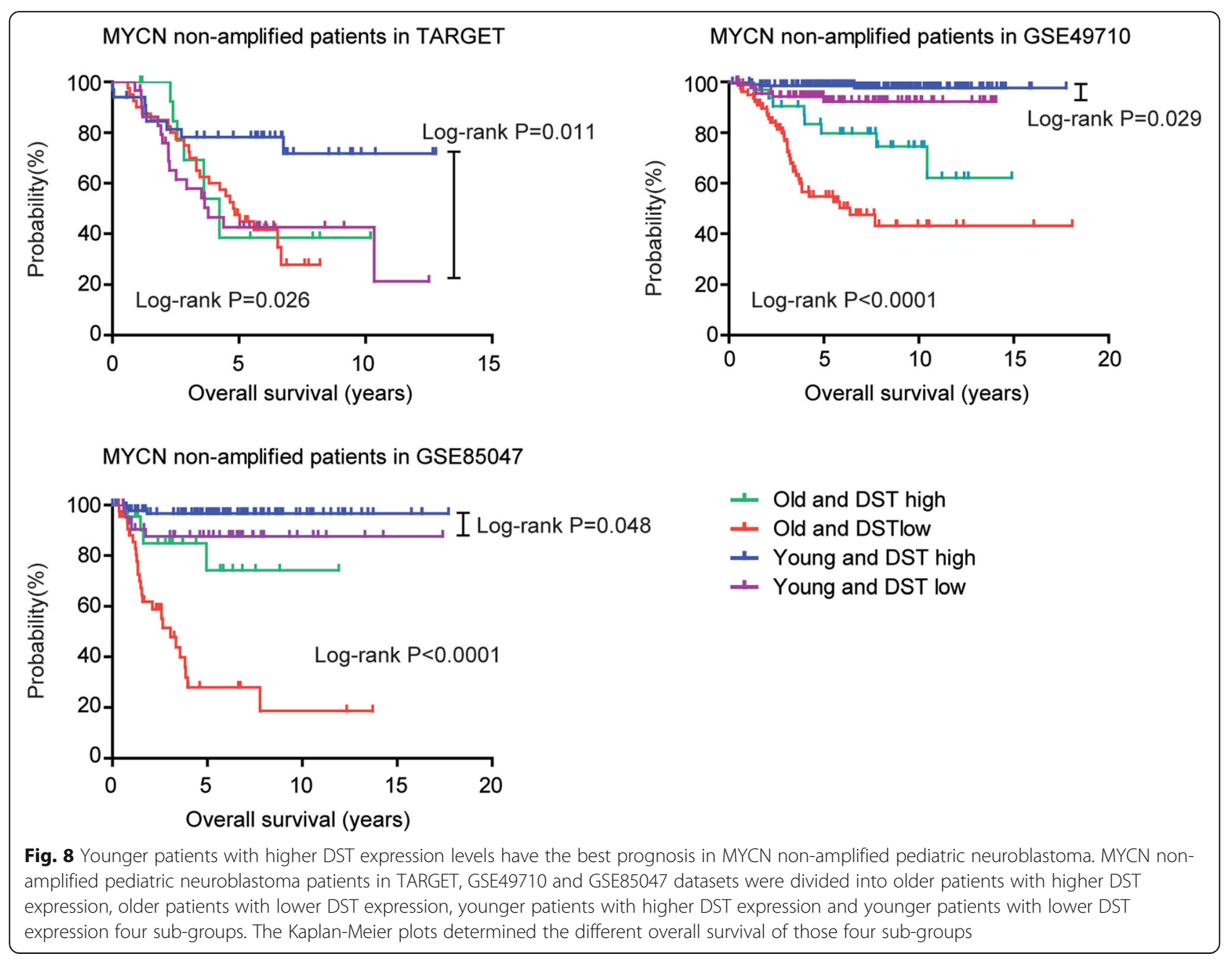

MYCN non-amplified pediatric neuroblastoma. And MYCN non-amplified pediatric neuroblastoma could be divided into several sub-groups by age and DST expression levels. However, there were some limitations in this study. The conclusions were generated from published datasets and lack of further validations. Therefore, functions and prognosis of DST in MYCN non-amplified neuroblastoma should be further studied.

\section{Conclusions}

MYCN non-amplified neuroblastoma is a heterogeneous disease and could be divided into sub-groups based on age or the expression levels of ALCAM, CACNA2D3, DST, EPB41L4A and KIF1B. MYCN non-amplified younger neuroblastoma patients with higher DST expression levels have the best clinical overall survival.

\section{Abbreviations}

TARGET: Therapeutically Applicable Research to Generate Effective Treatments; GEO: Gene Expression Omnibus; TCGA: The Cancer Genome Atlas; DAVID: The Database for Annotation, Visualization and Integrated
Discovery; KEGG: Kyoto Encyclopedia of Gens and Genomes; NRC: European Neuroblastoma Research Consortium; DST: Dystonin/BPAG1; HR: hazard ratio

\section{Acknowledgements}

We appreciate the generosity of the researches from TARGET and GEO groups for sharing the huge amount of data. We appreciate the permission from the Kanehisa laboratory to use the KEGG datasets.

\section{Authors' contributions}

HW.W and XR.W designed and performed data analysis. LP.X helped with the data analysis. HW.W wrote the manuscript. JZ and HC reviewed the manuscript and supervised the work. All authors read and approved the final manuscript.

\section{Funding}

This study was supported by grants from Fujian Maternity and Child Health Hospital (grant nos.YCXB 18-10 and YCXM 19-04). This study was also supported by Fujian Natural Science Foundation (grant nos.2020 J01337). But, the funders had no role in study design, data collection and analysis, decision to publish, or preparation of the manuscript.

\section{Availability of data and materials}

The datasets used and analyzed during the current study are available from the corresponding author on reasonable request and The TARGET datasets were downloaded from https://ocg.cancer.gov/. The GSE49710 and GSE85047 datasets were downloaded from https://www.ncbi.nlm.nih.gov/ geo/. 


\section{Declarations}

Ethics approval and consent to participate

Not applicable.

\section{Consent for publication}

Not applicable.

\section{Competing interests}

The authors declare that they have no competing interests.

Received: 5 February 2021 Accepted: 3 June 2021

Published online: 11 June 2021

\section{References}

1. Maris JM, Hogarty MD, Bagatell R, Cohn SL. Neuroblastoma. Lancet. 2007; 369(9579):2106-20.

2. Maris JM. Recent advances in neuroblastoma. N Engl J Med. 2010;362(23): 2202-11.

3. Cohn SL, Pearson AD, London WB, Monclair T, Ambros PF, Brodeur GM, et al. The international neuroblastoma risk group (INRG) classification system: an INRG task force report. J Clin Oncol. 2009;27(2):289-97.

4. Irwin MS, Park JR. Neuroblastoma: paradigm for precision medicine. Pediat Clin N Am. 2015:62(1):225-56.

5. Campbell K, Gastier-Foster JM, Mann M, Naranjo AH, Van Ryn C, Bagatell R, et al. Association of MYCN copy number with clinical features, tumor biology, and outcomes in neuroblastoma: a report from the Children's oncology group. Cancer. 2017;123(21):4224-35.

6. Look AT, Hayes FA, Shuster JJ, Douglass EC, Castleberry RP, Bowman LC, et al. Clinical relevance of tumor cell ploidy and $\mathrm{N}$-myc gene amplification in childhood neuroblastoma: a pediatric oncology group study. J Clin Oncol. 1991;9(4):581-91.

7. Durbin AD, Zimmerman MW, Dharia NV, Abraham BJ, Iniguez AB, WeichertLeahey $N$, et al. Selective gene dependencies in MYCN-amplified neuroblastoma include the core transcriptional regulatory circuitry. Nat Genet. 2018;50(9):1240-6.

8. Chan HS, Gallie BL, DeBoer G, Haddad G, Ikegaki N, Dimitroulakos J, et al. MYCN protein expression as a predictor of neuroblastoma prognosis. Clin Cancer Res. 1997;3(10):1699-706.

9. Valentijn LJ, Koster J, Haneveld F, Aissa RA, van Sluis P, Broekmans ME, et al. Functional MYCN signature predicts outcome of neuroblastoma irrespective of MYCN amplification. Proc Natl Acad Sci U S A. 2012;109(47):19190-5.

10. Wang H, Wang $X, X u$ L, Zhang J, Cao H. Prognostic significance of MYCN related genes in pediatric neuroblastoma: a study based on TARGET and GEO datasets. BMC Pediatr. 2020;20(1):314.

11. Felgenhauer J, Tomino L, Selich-Anderson J, Bopp E, Shah N. Dual BRD4 and AURKA inhibition is synergistic against MYCN-amplified and nonamplified neuroblastoma. Neoplasia. 2018:20(10):965-74.

12. Henssen A, Althoff K, Odersky A, Beckers A, Koche R, Speleman F, et al. Targeting MYCN-driven transcription by BET-Bromodomain inhibition. Clin Cancer Res. 2016;22(10):2470-81.

13. Puissant A, Frumm SM, Alexe G, Bassil CF, Qi J, Chanthery YH, et al. Targeting MYCN in neuroblastoma by BET bromodomain inhibition. Cancer Discov. 2013;3(3):308-23.

14. Park JR, Eggert A, Caron H. Neuroblastoma: biology, prognosis, and treatment. Hematol Oncol Clin North Am. 2010;24(1):65-86.

15. Ben-Aharon I, Elkabets M, Pelossof R, Yu KH, lacubuzio-Donahue CA, Leach $\mathrm{SD}$, et al. Genomic landscape of pancreatic adenocarcinoma in younger versus older patients: does age matter? Clin Cancer Res. 2019;25(7):2185-93.

16. Ma X, Liu Y, Liu Y, Alexandrov LB, Edmonson MN, Gawad C, et al. Pancancer genome and transcriptome analyses of 1,699 paediatric leukaemias and solid tumours. Nature. 2018;555(7696):371-6.

17. Su Z, Fang H, Hong H, Shi L, Zhang W, Zhang W, et al. An investigation of biomarkers derived from legacy microarray data for their utility in the RNAseq era. Genome Biol. 2014;15(12):523.

18. Rajbhandari P, Lopez G, Capdevila C, Salvatori B, Yu J, Rodriguez-Barrueco R, et al. Cross-cohort analysis identifies a TEAD4-MYCN positive feedback loop as the Core regulatory element of high-risk neuroblastoma. Cancer Discov. 2018;8(5):582-99.

19. Kanehisa M, Goto S. KEGG: Kyoto encyclopedia of genes and genomes. Nucleic Acids Res. 2000;28(1):27-30.
20. Kanehisa M. Toward understanding the origin and evolution of cellular organisms. Protein Sci. 2019:28(11):1947-51.

21. Kanehisa M, Furumichi M, Sato Y, Ishiguro-Watanabe M, Tanabe M. KEGG: integrating viruses and cellular organisms. Nucleic Acids Res. 2021;49(D1): D545-51.

22. Huang da W, Sherman BT, Lempicki RA: Systematic and integrative analysis of large gene lists using DAVID bioinformatics resources. Nat Protoc 2009; 4(1):44-57.

23. Huang da W, Sherman BT, Lempicki RA: Bioinformatics enrichment tools: paths toward the comprehensive functional analysis of large gene lists. Nucleic Acids Res 2009:37(1):1-13.

24. Wang H, Wang $X$, Xu L, Zhang J, Cao H. Prognostic significance of age related genes in patients with lower grade glioma. J Cancer. 2020;11(13): 3986-99.

25. Di Zenzo G, Della Torre R, Zambruno G, Borradori L. Bullous pemphigoid: from the clinic to the bench. Clin Dermatol. 2012;30(1):3-16.

26. Foureur N, Descamps V, Lebrun-Vignes B, Picard-Dahan C, Grossin M, Belaich S, et al. Bullous pemphigoid in a leg affected with hemiparesia: a possible relation of neurological diseases with bullous pemphigoid? Eur J Dermatol. 2001;11(3):230-3.

27. Cheng J, Liu HP, Hwang SL, Hsu LF, Lin WY, Tsai FJ. Dystonin/BPAG1 modulates diabetes and Alzheimer's disease cross-talk: a meta-analysis. Neurol Sci. 2019;40(8):1577-82.

28. Brick KE, Weaver CH, Savica R, Lohse CM, Pittelkow MR, Boeve BF, et al. A population-based study of the association between bullous pemphigoid and neurologic disorders. J Am Acad Dermatol. 2014;71(6):1191-7.

29. Poliakova K, Adebola A, Leung CL, Favre B, Liem RK, Schepens I, Borradori L. BPAG1a and b associate with EB1 and EB3 and modulate vesicular transport, Golgi apparatus structure, and cell migration in C2.7 myoblasts. PLoS One 2014;9(9):e107535.

30. Michael M, Begum R, Fong K, Pourreyrone C, South AP, McGrath JA, et al. BPAG1-e restricts keratinocyte migration through control of adhesion stability. J Invest Dermatol. 2014:134(3):773-82.

\section{Publisher's Note}

Springer Nature remains neutral with regard to jurisdictional claims in published maps and institutional affiliations.
Ready to submit your research? Choose BMC and benefit from:

- fast, convenient online submission

- thorough peer review by experienced researchers in your field

- rapid publication on acceptance

- support for research data, including large and complex data types

- gold Open Access which fosters wider collaboration and increased citations

- maximum visibility for your research: over $100 \mathrm{M}$ website views per year

At BMC, research is always in progress.

Learn more biomedcentral.com/submissions 